\title{
Complicações do estoma intestinal em pacientes em pós- operatório de ressecção de tumores de reto
}

\section{Complications of intestinal stoma in post-operative patients of rectal tumor resection}

\author{
Juliana Maximo da Costa' $\cdot$ Raquel de Souza Ramos ${ }^{2} \cdot$ Manassés Moura dos Santos $^{3}$ Daniela Ferreira da \\ Silva4 $\cdot$ Thays da Silva Gomes ${ }^{5} \cdot$ Renata Queiroz Batista $^{6}$
}

\begin{abstract}
RESUMO
A ocorrência de complicações relacionadas ao estoma intestinal tem impacto negativo na qualidade de vida do paciente estomizado. 0 presente estudo tem como objetivos: identificar e descrever as principais complicações relacionadas ao estoma intestinal em pacientes em pós-operatório de ressecção de tumores de reto. Estudo transversal, retrospectivo e descritivo, com técnica de coleta de dados por análise do prontuário físico e avaliação quantitativa dos mesmos. A coleta de dados ocorreu entre os meses de julho e novembro de 2016, e a análise quantitativa simples se deu a partir do programa Excel 2007. Nos resultados, apresentou-se a dermatite periestomal como a complicação mais encontrada (40,74\%), seguida de prolapso de estoma (29,62\%), necrose de mucosa estomal $(18,51 \%)$ e hérnia paracolostômica $(11,12 \%)$. Conclui-se que os resultados obtidos possibilitam o planejamento de ações direcionadas à prevenção das complicações e promoção do autocuidado do paciente estomizado. Dessa forma, faz-se necessária a reformulação do serviço de Estomaterapia, com quantitativo adequado de profissionais especializados garantindo qualidade na assistência prestada.
\end{abstract}

Palavras-chave: Estomia; Neoplasias Retais; Cuidados de Enfermagem; Avaliação em Enfermagem.

\section{ABSTRACT}

The occurrence of complications related to the intestinal stoma has a negative impact on the quality of life of the ostomized patient. The present study aims to identify and describe the main complications related to intestinal stoma in post-operative patients with resection of rectum tumors. Cross-sectional, retrospective and descriptive study, with data collection technique by physical chart analysis and quantitative evaluation. The data collection took place between July and November of 2016, and the simple quantitative analysis came from the program Excel 2007. The results show that peristomal dermatitis as the most frequent complication (40.74\%), followed by stoma prolapse (29.62\%), stomal necrosis (18.51\%) and paracolostomic hernia (11.12\%). It concludes that the results obtained allow the planning of actions aimed at the prevention of complications and promotion of selfcare of the stomized patient. Therefore, it possible to suggest the reformulation of the Stomatherapy service, encompassing the in-hospital and outpatient settings, with adequate numbers of specialized professionals to meet the demands of this clientele, guaranteeing quality in the assistance provided.

Keywords: Ostomy; Rectal Neoplasms; Nursing Care; Nursing Assessment.

${ }^{1}$ Enfermeira Especialista em Oncologia pelo Instituto Nacional do Câncer José Alencar Gomes da Silva (INCA). Enfermeira Pós-Graduada em Estomaterapia e em Clínica Cirúrgica pela Universidade Estadual do Rio de Janeiro, Brasil. E-mail: enfamaximo@gmail.com. Autor correspondente.

${ }^{2}$ Doutora em Enfermagem pela Universidade do Estado do Rio de Janeiro. Enfermeira do Hospital Universitário Pedro Ernesto e do Instituto Nacional do Câncer José Alencar Gomes da Silva (INCA). Rio de Janeiro, Brasil. E-mail: rramos@inca.gov.br.

${ }^{3}$ Mestre em Enfermagem pela Universidade do Estado do Rio de Janeiro. Enfermeiro do Instituto Nacional do Câncer José Alencar Gomes da Silva (INCA). Rio de Janeiro, Brasil. E-mail: manamoura@gmail.com.

${ }^{4}$ Mestre em Ciências do Cuidado em Saúde pela Escola de Enfermagem Aurora de Afonso Costa, Universidade Federal Fluminense (UFF). Enfermeira do Instituto Nacional do Câncer José Alencar Gomes da Silva (INCA). Rio de Janeiro, Brasil. E-mail: daniela.silva@inca.gov.br.

${ }^{5}$ Mestre em Cuidados de Enfermagem Fundamental pela Escola de Enfermagem Anna Nery, Universidade Federal do Rio de Janeiro (UFRJ). Enfermeira do Instituto Nacional do Câncer José Alencar Gomes da Silva (INCA). Rio de Janeiro, Brasil. Email: thaysenfalima@gmail.com.

${ }^{6}$ Enfermeira Especialista em Oncologia pelo Instituto Nacional do Câncer José Alencar Gomes d Silva (INCA). Rio de Janeiro, Brasil. E-mail: renata_qbatista@hotmail.com.

Não houve conflito de interesses durante a realização deste estudo. 


\section{INTRODUÇÃO}

No Brasil, o câncer colorretal é o $3^{\circ}$ tipo de câncer mais frequente na população. Segundo o Instituto Nacional do Câncer - INCA ${ }^{1-2}$, aparece como terceiro câncer mais incidente entre os homens, e segundo entre as mulheres. No âmbito mundial, o câncer colorretal apresenta o mesmo patamar de incidência. É considerada uma doença do "estilo de vida", com maior incidência em regiões com alto consumo de carne vermelha e produtos embutidos, obesidade e sobrepeso, além de pouca ingestão de fibras, frutas e verduras ${ }^{2}$.

O planejamento terapêutico realizado para o tratamento do câncer colorretal é orientado pela localização e extensão do tumor, sendo a ressecção cirúrgica o tratamento de escolha para tumores bem delimitados ${ }^{3}$. Tumores agressivos e localmente avançados necessitam de cirurgias mais extensas como a ressecção abdominoperineal (RAP) e a ressecção anterior de reto (RAR), a fim de garantir margem de segurança adequada e diminuir risco de recidivas locais ${ }^{4}$.

Para garantir o trânsito intestinal do paciente submetido às modalidades cirúrgicas para tratamento do câncer colorretal, a confecção de uma estomia intestinal pode ser necessária ${ }^{5}$. Esse tipo de procedimento não está livre de complicações, uma vez que seu sucesso depende de vários fatores, tais como: avaliação pré-operatória com demarcação do abdome, técnica cirúrgica e manuseio adequado dos dispositivos e materiais especiais.

A ocorrência de complicações no estoma intestinal pode se apresentar de forma precoce ou tardia. Dentre as complicações precoces, as mais comuns são: sangramento, isquemia, necrose, descolamento mucocutâneo, retração e edema ${ }^{6-7}$. Já entre as complicações tardias destacam-se a retração, estenose, prolapso de alça, fístula periestomal, abcesso periestomal, hérnia paraestomal ou paracolostômica e câncer ${ }^{6-8}$. A dermatite periestomal pode ocorrer precoce ou tardiamente, e pode ser causada por reação alérgica aos produtos utilizados, ou ainda pelo contato do efluente intestinal com a pele, causando um enorme transtorno no autocuidado do paciente estomizado ${ }^{7}$.

Tais complicações impactam de forma negativa no cotidiano da pessoa estomizada, principalmente no que refere-se à manutenção do autocuidado e bem-estar. Além disso, a ocorrência desses eventos pode causar maior tempo de internação, maiores gastos e maiores riscos para a reabilitação plena do paciente oncológico estomizado. $A$ atuação da equipe de enfermagem pode influenciar positivamente no processo de reabilitação do indivíduo estomizado, desde que a assistência seja contínua e iniciada precocemente 9 .

Como medidas preventivas, a localização adequada do estoma dentro dos limites do músculo reto abdominal através da demarcação pré-operatória, deve ser preconizada pelos serviços de cirurgia abdominal ${ }^{7}$. A realização da consulta de enfermagem como artifício para iniciar o desenvolvimento de confiança e vínculo, e ainda fornecer de forma precoce as orientações necessárias para facilitar o entendimento do paciente e sua família sobre sua nova condição, deve ser incentivada e adotada pelos serviços de saúde ${ }^{7}$. O registro de toda a assistência de enfermagem deve ser feito de forma clara e objetiva, garantindo a continuidade do atendimento e a comunicação entre os profissionais 6 .

Diante do que foi exposto, um estoma mal localizado em relação à sua posição na parede abdominal, ou proximidade de acidentes anatômicos, ferida operatória ou outras irregularidades corporais, ou ainda uma técnica cirúrgica mal executada podem causar inúmeras complicações gerando transtornos importantes para os pacientes submetidos às cirurgias abdomino-pélvicas ${ }^{8,10}$. Dessa forma, a não realização da demarcação abdominal, tida como procedimento fundamental na reabilitação do indivíduo estomizado, dentre outras ações no pré-operatório de cirurgias que resultam em confecção de estomia, tornamse um problema importante a ser discutido, visando reduzir complicações e facilitar o auto-cuidado do indivíduo submetido à confecção do estoma intetsinal ${ }^{10}$.

De posse das informações anteriores, foi definido como objeto de estudo as complicações dos estomas intestinais, e como objetivos da pesquisa: identificar e descrever as principais complicações relacionadas à confecção do estoma intestinal em pacientes submetidos à cirurgia de ressecção de tumores de reto. A escolha do objeto de estudo deu-se a partir da experiência na prática clínica oncológica durante o curso de especialização nos moldes de residência, com pacientes portadores das mais diversas estomias e com complicações importantes prejudicando seu bem-estar.

A justificativa deste estudo está centrada na possibilidade de fornecer subsídios para que o enfermeiro e equipe multiprofissional possam refletir sobre a prática profissional e sobre a necessidade de uma intervenção especializada em estomaterapia no pré-operatório, visando o bem-estar e um processo de reabilitação adequado, que traga qualidade de vida para as pessoas que convivem com um estoma intestinal qualificando, desta forma, a assistência prestada à este grupo específico de pacientes.

Como contribuições do estudo, pretende-se estimular a reflexão dos profissionais frente à temática do paciente estomizado e seus desdobramentos, e também da responsabilidade dos profissionais de saúde no registro correto das informações dos pacientes. Além disso, buscase fornecer informações que possam auxiliar no processo de reorganização do serviço de enfermagem, visando garantir assistência integral ao paciente estomizado.

\section{MÉTODO}

Trata-se de um estudo transversal, retrospectivo e descritivo, com técnica de coleta de dados por análise do prontuário físico de pacientes em pós-operatório de cirurgia 
para ressecção de tumores de reto, e avaliação quantitativa dos mesmos. Nesta pesquisa serão consideradas as variáveis do paciente, sexo e idade, além de variáveis relacionadas à cirurgia e às complicações cirúrgicas, como tipo de cirurgia e estomia, presença de complicações, tempo de complicação, e tipos de complicação associadas com o estoma intestinal.

Os critérios de inclusão elaborados para esse estudo foram: prontuários de pacientes adultos, admitidos na unidade para cirurgia eletiva de ressecção de tumor de reto, com confecção de estoma intestinal em 2015; e ainda com localização dos prontuários na unidade hospitalar elencada para estudo. Já os critérios de exclusão foram cirurgias sem confecção de estoma intestinal, prontuários pertencentes à outras unidades da instituição e ainda indivíduos submetidos à descompressão intestinal prévia.

A coleta de dados foi realizada no setor de arquivo médico de um Instituto especializado em tratamento oncológico na cidade do Rio de Janeiro, onde funciona o Serviço de Cirurgia Abdomino-pélvica responsável pelas principais cirurgias para tratamento do câncer de reto. 0 Comitê de Ética em Pesquisa da instituição em questão concedeu parecer final favorável para o presente estudo, sob n' 54679916.9.0000.5274.

Optou-se por rastrear todos os prontuários de pacientes maiores de idade submetidos à ressecção anterior de reto (RAR) ou ressecção abdômino-perineal de reto (RAP) no ano de 2015. A partir dos 98 prontuários selecionados, procedeu-se à coleta dos dados pertinentes para o estudo, com auxílio de uma ficha técnica elaborada para melhor agrupar as informações a serem analisadas.

Os prontuários foram analisados conforme disponibilidade no setor do arquivo médico, durante os meses de julho e novembro do ano de 2016. Dentre os 98 prontuários selecionados, 40 (40,81\%) não obedeciam aos critérios de inclusão previamente estabelecidos, sendo excluídos da análise documental posteriormente realizada. Dentre os motivos de exclusão dos 40 prontuários, $40 \%$ continham descrição de cirurgias sem confecção de estoma intestinal; $27,5 \%$ estavam localizados em outras unidades do hospital em estudo; e em 32,5\% haviam relatos de cirurgia prévia para descompressão intestinal.

Os 58 prontuários restantes foram submetidos à uma segunda análise, sendo esta quantitativa e descritiva. Inicialmente, 31 prontuários (53,44\%) não apresentavam registros de complicações por nenhum profissional de saúde, enquanto em 27 prontuários (46,55\%) haviam registros sobre a ocorrência de complicações relacionadas ao estoma intestinal. Durante a análise dos registros dessas complicações, foi possível observar que estes eram falhos e com poucas informações acerca do acompanhamento e desfecho das complicações, além da falta de comunicação/ interação dos profissionais da equipe multidisciplinar.

A análise quantitativa foi feita a partir do Excel 2007, de forma simplificada, relacionando as variáveis do estudo a fim de descrever as associações encontradas e compreender as características da população estudada. A análise descritiva se deu a partir da interpretação dos dados e associação com artigos já publicados, buscando contribuir para a melhoria da assistência prestada aos pacientes estomizados e garantir sua reabilitação após o tratamento oncológico.

\section{RESULTADOS}

\section{Caracterização da amostra}

Conforme os dados da tabela 1 , o número total de prontuários analisados foi de 58 ( $n=100 \%)$, sendo divididos em dois grupos: com complicações relacionadas ao estoma intestinal - GRUPO A, e sem complicações - GRUPO B.

Tabela 1. Total de prontuários utilizados para análise dos dados

\begin{tabular}{l|cc}
\hline \multicolumn{1}{c|}{ PRONTUÁRIOS PARA ANÁLISE } & N & \% \\
\hline COM COMPLICAÇÕES (Grupo A) & 27 & 46,55 \\
\hline SEM COMPLICAÇÕES (Grupo B) & 31 & 53,44 \\
\hline TOTAL & 58 & 100 \\
\hline
\end{tabular}

Fonte: Instituto Nacional do Câncer, 2016.

As principais complicações relacionadas ao estoma intestinal encontradas no material estudado foram: dermatite periestomal, prolapso de estoma, necrose de mucosa estomal e hérnia paracolostômica. Outras complicações também foram observadas, porém em menor ocorrência, conforme descrito na tabela 2.

Tabela 2. Complicações encontradas relacionadas ao estoma intestinal

\begin{tabular}{l|c}
\hline \multicolumn{1}{c|}{ COMPLICAÇÕES } & $\mathbf{n}$ \\
\hline PROLAPSO & 8 \\
\hline HÉRNIA PARACOLOSTÔMICA & 3 \\
\hline NECROSE & 5 \\
\hline DERMATITE & 11 \\
\hline GRANULOMA & 1 \\
\hline EDEMA & 1 \\
\hline HEMATOMA & 1 \\
\hline TRAUMA MECÂNICO & 1 \\
\hline DEISCÊNCIA PARCIAL DE SUTURA PARAESTOMAL & 4 \\
\hline DESCOLAMENTO MUCOCUTÂNEO & 1 \\
\hline ABCESSO PARACOLOSTÔMICO & 1 \\
\hline ALERGIA & 1 \\
\hline
\end{tabular}

Fonte: Instituto Nacional do Câncer, 2016.

A escolha da porção intestinal à ser exteriorizada pela parede abdominal para confecção do estoma pode variar de acordo com diversos fatores: condições anatômicas do paciente, extensão e localização do tumor, aderências em 
estruturas importantes, ou ainda a própria técnica cirúrgica. Houve predominância de confecção de colostomias frente às ileostomias, com 94,82\% e 5,18\% respectivamente.

Em relação à expectativa de tempo de permanência do estoma, $45,45 \%$ das colostomias foram confeccionadas de forma terminal, ou seja, sem previsão futura de reconstrução do trânsito intestinal.Já os estomas confeccionados apenas com o intuito de proteger a anastomose intestinal ocorreram em $100 \%$ das ileostomias, e em $54,54 \%$ das colostomias. De acordo com a tabela 3,100\% das ileostomias e 43,63\% das colostomias foram observadas no grupo A, e 56,36\% das colostomias localizadas no grupo B.

Tabela 3. Tipo de estoma relacionado com a ocorrência de complicações

\begin{tabular}{l|cc}
\hline \multicolumn{1}{c|}{ TIPO DE ESTOMA X COMPLICAÇÃO } & $\mathbf{n}$ & $\boldsymbol{\%}$ \\
\hline COLOSTOMIA & 55 & 100 \\
\hline GRUPO A & 24 & 43,63 \\
\hline GRUPOB & 31 & 56,36 \\
\hline ILEOSTOMIA & 3 & 100 \\
\hline GRUPO A & 3 & 100 \\
\hline GRUPO B & 0 & 0 \\
\hline
\end{tabular}

Fonte: Instituto Nacional do Câncer, 2016.

Em relação à técnica cirúrgica utilizada, ambos os grupos apresentaram valores similares. A ressecção anterior de reto (RAR), proposta em 1948 por Dixon, é a cirurgia de escolha para os tumores de reto superior e médio, com abordagem abdominal, sendo melhor executada após o advento das suturas mecânicas ${ }^{11}$. Já a cirurgia de Miles, como é conhecida a amputação abdomino-perineal (RAP), sofreu inúmeras modificações e contribuições técnicas ao longo dos anos, e ainda apresenta papel importante no tratamento do câncer retal de terço inferior, com invasão pélvica, metastático, entre outros ${ }^{12}$. Contudo, essa modalidade cirúrgica apresenta índices consideráveis de complicações graves como impotência sexual, bexiga neurogênica, esterilidade, complicações da colostomia, abscessos perineais e infecção do trato urinário ${ }^{12}$.

0 presente estudo revelou que 38 pacientes foram submetidos à modalidade RAR $(65,51 \%)$, enquanto apenas 20 foram operados com a técnica de RAP (34,48\%). Também foi observada a necessidade de realização de outros procedimentos associados ao tratamento cirúrgico do câncer de reto, seja por proximidade do câncer com outras estruturas, ou por conveniência, não colocando em risco o procedimento principal. 14 pacientes foram submetidos aos seguintes procedimentos: histerectomia bilateral, salpingectomia e ooforectomia bilateral (HTA, SOB), colecistectomia; prostatectomia e retirada de vesícula seminal, colpectomia posterior e nodulectomia hepática.

Relacionando o tipo de cirurgia com a ocorrência de complicações relacionadas ao estoma intestinal, foi constatado que a técnica cirúrgica com maior incidência de complicações foi a RAR (50\%), enquanto a RAP foi realizada em $40 \%$ dos pacientes com complicações. $\mathrm{Na}$ tabela 4, pode-se observar a relação entre os grupos A e B e a técnica cirúrgica realizada.

Tabela 4. Diferença entre os grupos A e B, com relação à técnica cirúrgica

\begin{tabular}{l|cc}
\hline & $\mathbf{n}$ & $\mathbf{\%}$ \\
\hline COM COMPLICAÇÃO (Grupo A) & 27 & 100 \\
\hline RAR & 19 & 70,37 \\
\hline RAP & 8 & 29,62 \\
\hline SEM COMPLICAÇÃO (Grupo B) & 31 & 100 \\
\hline RAR & 19 & 61,29 \\
\hline RAP & 12 & 38,7 \\
\hline
\end{tabular}

Fonte: Instituto Nacional do Câncer, 2016.

Não houve diferença significativa entre sexo no que se refere à ocorrência das complicações, sendo 44,44\% do sexo masculino e 55,55\% do sexo feminino. Quanto à idade, a faixa etária média foi de 60,03 anos, corroborando dados de outros estudos sobre a faixa etária de maior incidência no câncer de reto ${ }^{13-14}$.

Quanto aotempo de ocorrência das complicações, foi estabelecida como complicação precoce aquela ocorrida em até 2 semanas após o ato cirúrgico, e complicação tardia aquela ocorrida após esse tempo, sem limite posterior. 0 prolapso de estoma intestinal se deu $25 \%$ de forma precoce e $75 \%$ tardia, enquanto a hérnia paracolostômica se deu 100\% tardiamente, corroborando estudos anteriores ${ }^{15-16}$. A necrose de mucosa estomal aparece na literatura como uma complicação precoce, porém neste estudo se apresentou $60 \%$ de forma tardia. Já a dermatite periestomal teve sua ocorrência dividida basicamente pela metade, conforme descrito na tabela 5 , sendo considerada por muitos autores uma complicação que pode ocorrer tanto precoce como tardiamente.

Tabela 5. Tempo de ocorrência das complicações do estoma intestinal

\begin{tabular}{c|cc}
\hline & $\mathbf{n}$ & $\%$ \\
\hline PROLAPSO & 8 & 100 \\
\hline PRECOCE & 2 & 25 \\
\hline TARDIA & 6 & 75 \\
\hline HÉRNIA PARACOLOSTÔMICA & 3 & 100 \\
\hline TARDIA & 3 & 100 \\
\hline NECROSE DE MUCOSA ESTOMAL & 5 & 100 \\
\hline PRECOCE & 2 & 40 \\
\hline TARDIA & 3 & 60 \\
\hline DERMATITE PERIESTOMAL & 11 & 100 \\
\hline PRECOCE & 5 & 45,45 \\
\hline TARDIA & 6 & 54,54 \\
\hline
\end{tabular}

Fonte: Instituto Nacional do Câncer, 2016. 
De acordo com a tabela 6, a dermatite periestomal foi a complicação mais encontrada (40,74\%), seguida de prolapso de estoma (29,62\%), necrose de mucosa estomal $(18,51 \%)$ e hérnia paracolostômica $(11,12 \%)$.

Tabela 6. Relação entre o grupo A e as complicações encontradass

\begin{tabular}{l|cc}
\hline & $\mathbf{n}$ & $\boldsymbol{\%}$ \\
\hline GRUPO A & 27 & 100 \\
\hline DERMATITE & 11 & 40,74 \\
\hline PROLAPSO & 8 & 29,62 \\
\hline NECROSE & 5 & 18,51 \\
\hline HÉRNIA PARACOLOSTÔMICA & 3 & 11,12 \\
\hline
\end{tabular}

Fonte: Instituto Nacional do Câncer, 2016.

A complicação mais observada neste estudo foi a dermatite periestomal (40,74\%), com $100 \%$ de ocorrência em colostomias, fato evidenciado também por outros autores $7,8,15,16$. Granuloma, retração do estoma e estenose de anastomose coloanal foram outras complicações descritas nos pacientes que apresentaram dermatite periestomal, porém sem relação direta com o desenvolvimento da mesma.

A deiscência parcial de sutura paraestomal, ou descolamento mucocutâneo, também apareceu associada à dermatite periestomal, sendo uma complicação importante que pode propiciar o surgimento ou agravar quadros de dermatite periestomal já instalados, devido ao derramamento contínuo de fezes na pele, além do risco de retração do estoma e consequente peritonite ${ }^{7,14,16}$. Em relação à dermatite alérgica, foi evidenciado apenas 01 caso com registros de alergia ao adesivo microporoso do dispositivo coletor, sem mais informações.

As alterações dermatológicas mais frequentes nas dermatites são: eritema ou irritação, erosão, pústulas e até ulcerações além da dor intensa em queimação ${ }^{17}$, e podem ser classificadas em leve, moderada ou grave, dependendo do grau de comprometimento relativo à cor, umidade (coleção hídrica ou de pus) e à perda da integridade tecidual ${ }^{18}$. Também denominadas Dermatites Associadas à Incontinência (DAI), causam maiores danos à pele quando o efluente em questão é fecal, comparado ao efluente urinário, devido ao conteúdo bacteriano e à presença de enzimas que podem ser corrosivas na presença de um ph alcalino ${ }^{19}$. Dentre os fatores que influenciam no surgimento da DAl estão a umidade, o $\mathrm{PH}$ da pele, a colonização por microorganismos e o atrito excessivo ${ }^{19}$.

Tal complicação pode ser desencadeada por diversos fatores como: a falta de demarcação pré-operatória; o uso inadequado dos dispositivos e produtos tópicos (dermatite irritativa de contato); processos alérgicos aos materiais especiais (dermatite alérgica); trauma mecânico durante higiene excessiva e manipulação dos dispositivos; além da higiene precária7,15,18. Alterações de peso também podem influenciar no aparecimento de complicações periestomais, principalmente a dermatite irritativa de contato, sendo necessária readequação do dispositivo coletor ${ }^{16}$.

Desta forma, a presença da dermatite periestomal pode causar sofrimento e atraso no estabelecimento do autocuidado do paciente estomizado, devido à presença da dor e dificuldade de adesão aos materiais e dispositivos coletores. $\mathrm{O}$ enfermeiro estomaterapeuta (ET) deve levar em consideração os aspectos físicos, sociais e emocionais do paciente estomizado, a fim de melhor compreender sua realidade e adequar suas condutas ${ }^{20}$.

O prolapso do estoma intestinal foi observado em 29,62\% dos pacientes com complicações, com faixa etária entre 49 e 75 anos, e somente em colostomias. É considerada uma complicação tardia, fato observado em $75 \%$ dos prolapsos neste estudo, e que apresenta grande impacto no autocuidado e reabilitação do paciente, pois ocorre exteriorização exacerbada da alça intestinal através da parede abdominal, prejudicando a limpeza e adequação do dispositivo coletor ${ }^{16}$.

Houve associação do prolapso de estoma com 02 casos de hérnia paracolostômica, corroborando a relação entre as 02 complicações descritas em outros estudos, tendo como fatores de ordem comum a fragilidade do tônus muscular relacionada à idade e sedentarismo, técnica cirúrgica inadequada, obesidade e aumento da pressão intra-abdominal ${ }^{10-21-22}$. As causas mais comuns descritas na literatura são a abertura excessiva da parede abdominal durante o ato operatório, deixando o estoma "frouxo", e o aumento da pressão intra-abdominal devido à diversos fatores: esforço abdominal, vômitos intensos, tosse, entre outros $^{10-15-21}$

$\mathrm{Na}$ vigência desta complicação, existe o risco de trauma mecânico durante manipulação do estoma e seus dispositivos, o que foi evidenciado em 01 dos prontuários estudados, no qual constava o registro de necrose, relatado pelo ET. Outros estudos também associam a dermatite periestomal ao prolapso de alça intestinal, pois dependendo da extensão do prolapso e do manuseio do dispositivo coletor, pode haver contato contínuo do efluente intestinal com a pele, reduzindo a aderência da bolsa, favorecendo o aparecimento de dermatites importantes ${ }^{22}$.

A intervenção cirúrgica de urgência com secção da alça ou possível realocação do estoma na parede abdominal, está indicada diante de sinais de isquemia e sofrimento de alça ou quando associada com hérnia paracolostômica volumosa ${ }^{10}$. Em situações leves, o ET capacitado pode recorrer à algumas condutas:

- Redução manual (manobra de reinserção manual da alça intestinal associada à compressas frias, sendo um artifício considerado paliativo);

- Aplicação local de gelo ou solução glicosada em compressas por 30 minutos (sempre protegendo a alça intestinal com compressas limpas e úmidas); 
- Infiltração submucosa de solução de lidocaína e soro fisiológico, o que auxilia na redução do edema e facilita a manipulação da porção intestinal ${ }^{10-15}$.

A recidiva é comum, devido às suas possíveis causas ${ }^{15}$. Desta forma, orientações sobre restrição de atividades que demandam grande esforço físico e conscientização de familiares e cuidadores sobre a necessidade e importância da mudança de estilo de vida, devem ser ofertadas precocemente?

A hérnia paracolostômica, ou paraestomal, foi evidenciada em $11,11 \%$ dos pacientes, com faixa etária entre 60 e 69 anos, estando somente associada ao prolapso de estoma intestinal, como descrito anteriormente.

Assim como o prolapso, a hérnia paracolostômica causa problemas graves no cotidiano do indivíduo estomizado. Se muito volumosa, pode causar dores intensas, obstrução, dificuldade na adaptação do dispositivo coletor, sofrimento psicológico e isolamento social, sendo indicativa à cirurgia ${ }^{15}$. Os fatores de risco para o desenvolvimento desta complicação incluem: idade avançada, aumento de peso, presença de outras hérnias e confecção do estoma fora dos limites do músculo reto abdominal ${ }^{15-21}$.

A utilização de cintas abdominais modificadas, ou a indicação de dispositivo coletor com placa convexa e cinto adaptável podem auxiliar na contenção da musculatura abdominal ${ }^{10-21}$. Durante a consulta de enfermagem, o ET deve avaliar e orientar a melhor posição para colocação do dispositivo coletor, sentado, deitado ou em pé, considerando a independência, conforto e bem-estar do paciente ${ }^{10}$.

A indicação de tratamento da hérnia paracolostômica pode variar de acordo com suas dimensões e com a influência exercida sobre o cotidiano e bem-estar do paciente estomizado, pois pode causar dores intensas, risco de obstrução intestinal e isolamento social devido às alterações físicas. Hérnias de pequeno volume podem ser tratadas com sutura local ou através de procedimento videolaparoscópico. Porém hérnias volumosas necessitam de intervenções maiores, com possível realocação do estoma intestinal na parede abdominal e uso de telas confeccionadas com material sintético, para corrigir falhas na aponeurose $\mathrm{e}^{21-23}$.

0 relato de necrose de mucosa estomal foi encontrado em $18,51 \%$ dos prontuários estudados, $40 \%$ masculinos e $60 \%$ femininos. Sua descrição nos registros estudados se deu como "esfacelo na mucosa estomal",e em 02 prontuários estavam associados ao descolamento mucocutâneo, sem informações sobre condutas específicas.

A necrose é decorrente de outra complicação, a isquemia, resultante da manipulação inadequada e falta de irrigação sanguínea para a alça intestinal exteriorizada ${ }^{21}$. Caracteriza-se por mudança na coloração da mucosa intestinal, que pode apresentar tons arroxeados ou ate mesmo acinzentados ou enegrecidos. Quando a isquemia persiste sem que haja intervenção cirúrgica, o tecido estomal morre, adquirindo coloração amarelada, marrom ou preta e consistência macia e flácida ${ }^{15}$. 0 tratamento da isquemia e necrose isquêmica é cirúrgico, podendo ser indicado à laparotomia para ressecção do seguimento necrosado e confecção de novo estoma ${ }^{21}$.

Outras complicações foram observadas associadas às principais complicações já abordadas. Porém, não haviam registros específicos ou ainda descrição minuciosa da evolução dos casos. Dentre as complicações menos frequentes o descolamento mucocutâneo, descrito como deiscência parcial de sutura paraestomal, apareceu em 04 relatos do ET, sendo uma complicação importante no manejo da estomia, pois contribui para o desenvolvimento de dermatites graves ${ }^{14}$.

No que diz respeito às condutas específicas do ET frente às complicações observadas, como indicação de materiais e cuidados especiais, o principal tratamento para dermatite periestomal descrito nos prontuários utilizados neste estudo foi o protetor cutâneo spray, sendo também utilizado o pó para estomia e placas com pectina. Os dispositivos utilizados como barreira protetora de pele podem ter diversas apresentações (placa, disco, anel, pasta, massa ou pó), e sua composição pode variar: carboximetilcelulose sódica (CMC), gelatina, pectina, entre outros ${ }^{24}$.

Essas substâncias têm a finalidade de proteger a pele periestomal da ação do efluente, devendo manter suas condições fisiológicas, tanto de temperatura como umidade e pH. Permite também a reparação das lesões quando utilizada sobre a pele lesada, devendo ser hipoalergênica ${ }^{24}$. A utilização de grânulos em pó (redução da umidade), dispositivos coletores especiais (convexo, duas peças) e outros materiais específicos, deve ser indicada pelo $\mathrm{ET}^{14-15-19}$.

Para o descolamento mucocutâneo foi indicado o uso de alginato de cálcio em fitas para preencher as cavidades formadas, barreira cutânea em spray, placas e pastas com pectina. A indicação do alginato de cálcio é valida, pois esta cobertura auxilia no desbridamento autolítico, promove agregação plaquetária (hemostático), diminui o exsudato e o odor da ferida (bacteriostático), e pode ser usado em feridas cavitárias, tunelizantes, com ou sem infecção ${ }^{24}$. Não havia maiores informações referentes ao tratamento tópico, como também não houve relato de agravamento dos casos.

Dentre os relatos sobre o tratamento dos casos de prolapso, houve somente um registro específico do ET: uso de dispositivo coletor de duas peças para melhor adaptar a alça intestinal exteriorizada. Contudo, não havia registro sobre as orientações realizadas ao paciente sobre manipulação desse dispositivo. 0 restante das condutas frente à ocorrência de prolapso e hérnia paracolostômica eram médicas, com indicação de reconstrução do trânsito intestinal (RTI) ou decolostomia. 0 uso de cinta abdominal adaptada ou ainda o procedimento de redução manual não foram citados.

Sobre o tratamento da necrose estomal, 02 registros feitos pelo ET indicavam "tratamento conservador", 
entretanto, sem especificar outras condutas ou orientações fornecidas ao paciente. Outros 02 registros estavam associados à ocorrência do descolamento mucocutâneo, indicando o uso do alginato de cálcio e protetores cutâneos como já descrito, contudo, não estava evidente a indicação dos materiais para o tratamento da necrose.

O ET tem como prioridade avaliar minuciosamente a extensão da necrose estomal, e lançar mão das técnicas de escarificação e desbridamento, quando indicadas, além do uso de coberturas específicas. 0 uso de dispositivos coletores transparentes no pós-operatório imediato é preconizado, pois permite visualização constante do aspecto dos estomas intestinais, facilitando a detecção precoce de complicações ${ }^{15-16-21}$.

\section{DISCUSSÃO}

A ocorrência de complicações relacionadas ao estoma intestinal pode ser minimizada. Para isso, deve ser realizado um planejamento que englobe o pré, intra e pós-operatório, além de toda a equipe multidisciplinar. A falta de experiência da equipe cirúrgica, incluindo a execução do procedimento por médicos residentes, e o local inadequado para exteriorização do estoma (falta de demarcação pré-operatória) são consideradas as principais causas de falência no sucesso da confecção do estoma intestinal ${ }^{7-8-21}$.

A atuação especializada do ET frente às complicações relacionadas ao estoma intestinal mostra-se indispensável, visando a reabilitação plena e a promoção da qualidade de vida do paciente cirúrgico. Dentre as atribuições do enfermeiro especialista em Estomaterapia, podemos dizer que a demarcação do local de confecção do estoma no pré-operatório imediato é o principal cuidado à ser implementado na assistência de enfermagem ao paciente submetido à confecção de um estoma intestinal ou urinário, visto que este procedimento pode reduzir a ocorrência de complicações preveníveis, como a hérnia paracolostômica, o prolapso de alça intestinal e a dermatite periestomal ${ }^{10}$.

Segundo a Sociedade Brasileira em Estomaterapia (SOBEST), a visita pré-operatória, a demarcação e orientações pertinentes (procedimento cirúrgico, estoma, dispositivos), além do acompanhamento pós-operatório (domiciliar ou ambulatorial), são atribuições do enfermeiro estomaterapeuta, e contribuem para a detecção precoce de complicações, além de garantir segurança ao paciente estomizado ${ }^{20}$.

A avaliação do nível de compreensão do paciente e seu familiar/cuidador deve ser realizada no primeiro atendimento, pois orientações insuficientes ou mal compreendidas podem afetar os meios de enfrentamento do paciente diante a sua nova condição de estomizado, resolução das complicações e formas de prevenção das mesmas. A atuação da equipe de enfermagem tem papel fundamental na implantação de estratégias em educação em saúde, promoção do bem-estar e reabilitação do indivíduo estomizado ${ }^{7}$.
Contudo, a área de atuação do ET vai além das orientações técnicas, devendo abranger também a perspectiva de reabilitação social e emocional do paciente estomizado. As alterações físicas e emocionais trazidas pela confecção do estoma intestinal e suas complicações, podem acarretar prejuízos importantes na vida social e pessoal do paciente estomizado, gerando sofrimento e possível abandono do autocuidado. A construção de um espaço seguro, de escuta e acolhimento, deve ser iniciada ainda no pré-operatório, de modo a garantir total entendimento por parte do paciente acerca das mudanças que irão acontecer, diminuindo a ansiedade ${ }^{9}$.

De acordo com o que já foi discutido, fica evidente a necessidade de implementação de um serviço especializado em Estomaterapia, no âmbito hospitalar e ambulatorial, e adequado a cada realidade institucional, com o intuito de proporcionar à esta clientela específica, um atendimento integral e de qualidade. 0 recrutamento de profissionais especializados e o planejamento de estratégias para assegurar a atuação do ET sem sobrecarregar outras atividades devem ser pensados o quanto antes, pois suas ações são indispensáveis no manejo dos pacientes cirúrgicos, estomizados e suas complicações.

Durante coleta e análise dos dados, foi possível observar que os registros da equipe de saúde eram falhos, não havendo: continuidade nas informações referentes ao tratamento, descrição completa das características do estoma e/ou da complicação apresentada, evolução do problema e desfecho com consequente alta do atendimento, caracterizando falha grave na qualidade da assistência de enfermagem.

Alguns possíveis motivos para a falta de continuidade e registros específicos seriam: número insuficiente de enfermeiros especialistas para 0 atendimento desta clientela; falha no encaminhamento para o ambulatório de Estomaterapia por parte do profissional médico ou enfermeiro que acompanha o paciente durante sua internação; ou ainda a não aderência dos usuários por desconhecimento sobre a importância da atuação deste profissional frente às complicações, reabilitação e autocuidado.

\section{CONCLUSÃO}

Os resultados obtidos com este estudo mostram a necessidade de constante discussão sobre o cuidado voltado ao paciente estomizado, e manejo das possíveis complicações inerentes ao estoma intestinal. A relevância dos dados encontrados está pautada na possibilidade de planejamento de ações direcionadas à prevenção de complicações e promoção do autocuidado, visto que a maioria das complicações observadas podem ser prevenidas através de assistência especializada.Além disso, a caracterização das complicações encontradas auxilia o profissional de saúde à desenvolver um olhar crítico sobre o indivíduo estomizado, com maiores chances de detecção precoce de complicações importantes. 
Dessa forma,o reconhecimento precoce das complicações e a construção do saber acerca das peculiaridades desta clientela, materiais especiais utilizados no tratamento das complicações relacionadas ao estoma e outras demandas, tornam-se ações indispensáveis na prática do profissional enfermeiro, seja estomaterapeuta ou não, devido à ocorrência comum em diversos ambientes de saúde.

A atuação de profissionais especializados e atualizados torna-se primordial na assistência aos pacientes em pósoperatório de ressecção de tumores de reto, e outras cirurgias abdomino-pélvicas. Portanto, verifica-se a necessidade de reformulação do serviço de Estomaterapia, com quantitativo adequado de profissionais especializados, focados em atender as demandas dos pacientes estomizados (e outros) com qualidade, no ambiente hospitalar e ambulatorial.

Além disso, é importante salientar que o trabalho conjunto da equipe multiprofissional se faz necessário. A conscientização de toda a equipe, principalmente dos profissionais médicos, sobre a real necessidade de atividades específicas do enfermeiro estomaterapeuta, como a realização da demarcação pré-operatória, é primordial para um cuidado integral a essa clientela, objetivando reduzir complicações pós-operatórias, promover a reabilitação e manutenção do autocuidado.

Como já pontuado, a continuidade dos registros encontrados feitos pela equipe de saúde acerca das complicações relacionadas ao estoma intestinal foram falhos, o que dificultou a análise descritiva de forma mais aprofundada, quanto ao desenrolar do processo de evolução dessas complicações. Quanto à escassez de informações encontradas nos registros em prontuário, pode-se sugerir que o quantitativo de profissionais capacitados para este atendimento é inferior ao necessário para execução das atividades inerentes ao estomaterapeuta, prejudicando a continuidade do tratamento dos pacientes, e também a comunicação entre os membros da equipe multiprofissional.

Sugere-se a reformulação do serviço de Estomaterapia, abrangendo o ambiente intra-hospitalar e ambulatorial, de forma a alcançar todo o período transoperatório do paciente submetido às modalidades cirúrgicas que possam originar um estoma. A construção e adoção de formulários, impressos e escalas de avaliação próprios para a área de Estomaterapia auxilia no processo de tomada de decisão e direciona o cuidado de forma individualizada, garantindo qualidade no atendimento ao público estomizado. Com isso, o planejamento e execução dos cuidados de enfermagem tornam-se devidamente aplicáveis durante a prática clínica, garantindo qualidade no atendimento e reconhecimento profissional.

\section{REFERÊNCIAS}

1. Instituto Nacional de Câncer José Alencar Gomes da Silva. Coordenação de Prevenção e Vigilância Estimativa 2014: Incidência de Câncer no Brasil / Instituto Nacional de Câncer
José Alencar Gomes da Silva, Coordenação de Prevenção e Vigilância. Rio de Janeiro: INCA [Internet]. 2014 [acesso em 24 jan 2016]. Disponível em: http://www.inca.gov.br/ rbc/n_60/v01/pdf/11-resenha-estimativa-2014-incidenciade-cancer-no-brasil.pdf.

2. Instituto Nacional de Câncer José Alencar Gomes da Silva. Coordenação de Prevenção e Vigilância Estimativa 2016: Incidência de Câncer no Brasil / Instituto Nacional de Câncer José Alencar Gomes da Silva, Coordenação de Prevenção e Vigilância. Rio de Janeiro: INCA [Internet]. 2016 [acesso em 24 jan 2016]. Disponível em: http://www.inca.gov.br/wcm/ dncc/2015/estimativa-2016.asp.

3. Ministério da Saúde (Brasil). Portaria n 601, de 26 de junho de 2012. Aprova as Diretrizes Diagnósticas e Terapêuticas do Câncer de Cólon e Reto. Diário Oficial da União 26 jun 2012 [Internet]. 2012 [acesso em 26 jan 2016]. Disponível em: http://bvsms.saude.gov.br/bvs/saudelegis/sas/2012/ prt0601_26_06_2012.html.

4. Ramos JR, Mesquita RM, Valory EA, Santos F. Tratamento cirúrgico do câncer da porção distal do têrço inferior do reto pela ressecção anterior ultrabaixa e interesfinctérica com anastomose coloanal por videolaparoscopia. Rev bras. colo-proctol. [Internet]. 2009 [acesso em 26 jan 2016]. Disponível em: http://www.scielo.br/scielo.php?script=sci_ arttext \&pid=S0101-98802009000300005 \&lng=en.

5. Sarmento BJ, Campoli PM, Brito AM, Silva OQ, Santana Filho JB, Barreto PA et al. Papel da Excisão Total do Mesorreto no Tratamento Multimodal do Câncer de Reto. Revista Brasileira de Cancerologia [Internet]. 2008 [acesso em 27 jan 2016]; 54(1): 87-96. Disponível em: http://www.inca.gov.br/ rbc/n_54/v01/pdf/revisao_6_pag_87a96.pdf.

6. Rocha JJR. Estomas intestinais (ileostomias e colostomias) e anastomoses intestinais. Simpósio. Fundamentos em clínica cirúrgica. Medicina (Ribeirão Preto) [Internet]. 2011 [acesso em 7 fev 2016] ; 44(1): 51-6. Disponível em: http://revista.fmrp.usp. br/2011/vol44n1/Simp5_Estomas\%20intestinais.pdf.

7. Yamada BFA, Cesaretti IUR, Marcondes MG, Morais JF, Prado AAB. Ocorrência de Complicações no Estoma e Pele Periestoma - estudo retrospectivo. Rev Estima [Internet]. 2003 [acesso em 7 fev 2016]; 1(3):16-24. Disponível em: http://www.revistaestima.com.br/index.php?option=com_ content \&amp;view=article\&amp;id=287\%3A.

8. Santos CHM, Bezerra MM, Bezerra FMM, Paraguassú BR. Perfil do Paciente Ostomizado e Complicações Relacionadas ao Estoma. Rev bras Coloproct [Internet]. 2007 [acesso em 9 fev 2016]; 27(1): 016-019. Disponível em: http://www.scielo.br/ pdf/rbc/v27n1/a02v27n1.pdf.

9. Nascimento DC, Chagas CC, Souza NVDO, Marques GS, Rodrigues FR, Cunha CV. Experiência Cotidiana: a Visão da Pessoa com Estomia Intestinal, Rev Estima [Internet]. 2016 [acesso em 04 jan 2017]; 14(4):183-192. Disponível em: https://www.revistaestima.com.br/index.php/estima/article/ view/431.

10. Meirelles CA, Ferraz CA. Avaliação da qualidade do processo de demarcação do estoma intestinal e das intercorrências tardias em pacientes ostomizados. Rev Latino-am Enfermagem [Internet]. 2001 [acesso em 05 jan 2016]; 9(5):32-8. Disponível em: http://dx.doi.org/10.1590/S010411692001000500006. 
11. Oliveira JC. Anastomose colo-retal por grampeamento duplo. Arc Cat Med/ [Internet]. 1997 [acesso em 06 fev 2016]; 26(14):9-13. Disponível em: http://www.acm.org.br/revista/pdf/ artigos/36.pdf.

12. Cruz GMG, Ferreira RMRS, Neves PM. Amputação Abdominoperineal: Uma Cirurgia Fora de Moda? - Estudo Retrospectivo de 135 Cirurgias Realizadas ao Longo de Quatro Décadas. Rev bras Coloproct [Internet]. 2004 [acesso em 09 fev 2016]; 24(2): 103-118. Disponível em: http://www. sbcp.org.br/revista/nbr242/P103_118.html.

13. Rêgo AGS, Borges ICV, Valença RJV; Teles JBM, Pinto LSS. Câncer Colorretal em Pacientes Jovens. Rev Bras Cancerologia [Internet]. 2015 [acesso em 18 dez 2016]; 58(2): 173-180. Disponível em: http://www1.inca.gov.br/rbc/n_58/v02/ pdf/07_artigo_cancer_colorretal_pacientes_jovens.pdf.

14. Diniz IV, Ancelmo MN, Soares RAS, Santos IBC, Oliveira MJGS. Problema de Pele em Paciente Estomizada: Relato de Caso. Rev bras cien saúde [Internet]. 2013 [acesso em 18 dez 2016]; v.17, n.4 (2013): 371-378. Disponível em: http://periodicos. ufpb.br/ojs/index.php/rbcs/issue/view/1440.

15. Cruz GMG, Constantino JRM, Chamone BC, Andrade MMA, Gomes DMBM. Complicações dos estomas em câncer colorretal: revisão de 21 complicações em 276 estomas realizados em 870 pacientes portadores de câncer colorretal. Rev bras. colo-proctol. [Internet]. 2008 [acesso em 07 fev 2016]; 28(1): 50-61. Disponível em: http:// www.scielo.br/scielo.php?script=sci_arttext \&pid=S010198802008000100008\&lng=en.

16. Barata AM. Considerações sobre estomas entéricos parte ii - construção e complicações. Revista portuguesa de coloproctologia [Internet]. 2009 [acesso em 14 fev 2016]; maio/ago (2): 24-27. Disponível em: http://spcoloprocto.org/ uploads/rpcol_maio_agosto_2009_pags_24_a_27.pdf.

17. Cunha CV, Ferreira D, Nascimento D, Felix F, Cunha P, Penna LHG. Artigo de Revisão - Dermatite associada à incontinência em idosos: caracterização, prevenção e tratamento. Rev Estima [Internet]. 2015 [acesso em 20 dez 2016]; 13(3). Disponível em: https://www.revistaestima.com.br/index.php/estima/article/ view/218.

18. Silva IS. Prevenção de dermatite em pele periestoma na estomia intestinal. Bahia, 2015. 10p. Projeto de pesquisa apresentado à disciplina de Metodologia da Pesquisa I do Curso de Enfermagem Dermatológica da Atualiza [Internet]. 2015 [acesso em 04 jan 2017]. Disponível em: http://bibliotecaatualiza.com. br/arquivotcc/ED/ED01/SILVA-itana.pdf.

19. Soares, VA, Estrela, FM, Rey, MD, Rocha, TA, Macedo, F, Moreira, BSG, Gomes, NP. Manejo da dermatite associada à incontinência: utilização de creme com função barreira. Rev Enf Atual [Internet]. 2013 [acesso em 03 jan 2017]; 65(3):2628. Disponível em: http://inderme.com.br/03-05.html.

20. Associação Brasileira de Estomaterapia. Competências do Enfermeiro Estomaterapeuta Ti SOBEST ou do Enfermeiro Estomaterapeuta. SOBEST [Internet]. 2009 [acesso em 07 jan 2017]. Disponível em: http://www.sobest.org.br/texto/11.

21. Torres CBD, Andrade EMLR, Ribeiro FMS, Neta FCCG, Luz MHBA. Qualidade de vida de pessoas estomizadas: revisão integrativa. Rev Enferm UFPI [Internet]. 2015 [acesso em 08 fev 2016]; 4(1):117-22. Disponível em: http://www.ojs.ufpi. br/index.php/reufpi/article/view/2242.
22. Dias MAS. Complicações das ostomias urinárias e digestivas. Mestrado Integrado em Medicina. Faculdade de Medicina. Universidade do Porto [Internet]. 2010 [acesso em 15 fev 2016]. Disponível em: https://repositorio-aberto. up.pt/bitstream/10216/53608/2/Complicaes\%20das\%20 ostomias\%20urinrias\%20e\%20digestivas.pdf.

23. Carvalho CG, Vale CEP, Castro JR. PC. Experiência inicial no tratamento das hérnias paraestomais. Rev bras Coloproct [Internet]. 2008 [acesso em 04 jan 2017]; 28(2): 251-256. Disponível em: http://www.scielo.br/scielo.php?script=sci_ arttext\&pid=S0101-98802008000200017.

24. Domansky RC. Ostomias: Conhecendo a Composição das Barreiras Protetoras de Pele. Rev Estima [Internet]. 2003 [acesso em 09 jan 2017]; 1(2). Disponível em: https://www.revistaestima.com.br/ index.php/estima/article/view/. 